\title{
Conhecimento etnoentomológico dos moradores do município de Buriticupu, Maranhão, Brasil
}

\author{
Andreson de Carvalho Alves ${ }^{1 *}$ \\ Amália dos Anjos Beims ${ }^{1}$ \\ Elisama de Melo Alencar ${ }^{1}$ \\ Franciane Alencar de Lima ${ }^{1}$ \\ Kaires Mayane Araújo da Silva ${ }^{1}$ \\ EIson Silva de Sousa ${ }^{2}$ \\ Reinaldo Lucas Cajaiba ${ }^{2}$
${ }^{1}$ Instituto Federal de Educação, Ciência e Tecnologia do Maranhão, Campus Buriticupu Rua Deputado Gastão Vieira, 1000, CEP 65.393-000, Buriticupu - MA, Brasil
${ }^{2}$ Laboratório de Ecologia e Conservação Instituto Federal de Educação, Ciência e Tecnologia do Maranhão, Campus Buriticupu
* Autor para correspondência andreson.alves@acad.ifma.edu.br

Submetido em 26/08/2018

Aceito para publicação em 19/02/2019

\section{Resumo}

Os insetos desempenham funções vitais nos ecossistemas em que ocorrem, porém são muitas vezes percebidos de forma negativa pelo homem. Essa percepção é domínio da etnoentomologia, que, de modo geral, tem contribuído para unir os conhecimentos científicos e os saberes tradicionais. Tendo isso em vista, este estudo teve como objetivo avaliar a percepção da população do município de Buriticupu, Maranhão, sobre os insetos. Foram entrevistados 107 moradores, por meio do emprego de formulários semiestruturados. Os resultados demonstraram que o termo "inseto" foi associado tanto a animais que pertencem à classe Insecta como a outros grupos taxonômicos e que a maioria dos entrevistados apresenta um sentimento negativo em relação aos insetos. A percepção negativa a respeitos dos insetos, aliada à ausência do conhecimento, pela comunidade, acerca da importância deles na natureza, fortalece ações agressivas contra tais animais. As informações obtidas neste estudo devem ser usadas em palestras e em projetos de extensão que venham a informar a população sobre a importância dos insetos.

Palavras-chave: Conhecimento tradicional; Etnobiologia; Percepções

\section{Abstract}

Ethnoentomological knowledge of the inhabitants of the municipality of Buriticupu, Maranhão, Brazil. Insects play vital roles in the ecosystems where they occur but are often perceived negatively by humans. This perception is within the domain of ethnoentomology that, in general, has contributed to uniting scientific and traditional knowledge. The objective of this study was to evaluate the perception the population of the 
municipality of Buriticupu, Maranhão, has on insects. One hundred and seven residents were interviewed using semi-structured forms. The results demonstrated that the term "insect" was both associated to animals belonging to class Insecta and other taxonomic groups and that most of the interviewees had a negative feeling about insects. In the community, the negative perception of insects and the lack of knowledge about the importance of insects in nature contribute to aggressive actions against these animals. The information obtained in this study should be used in lectures and extension projects that inform the population about the importance of insects.

Key words: Ethnobiology; Perceptions; Traditional knowledge

\section{Introdução}

Os insetos constituem o maior grupo de animal existente, ocupando espaço em quase todos os ecossistemas. São componentes-chave na estrutura e no funcionamento dos ecossistemas, apresentando importantes papéis tróficos nas redes alimentares, seja como predadores ou fitófagos (NITZU et al., 2008; QODRI et al., 2016), controlando insetos pragas da agricultura (HOLANDA-NETO, 2002) e ervas daninhas (BOHAN et al., 2011; CAJAIBA et al., 2018). Muitas espécies desempenham, ainda, a função de dispersão de sementes e de decomposição de matéria orgânica, o que contribui para melhoria das propriedades físico-químicas do solo e ajuda no crescimento das plantas (CAJAIBA et al., 2017a; 2017b).

A grande diversidade e distribuição dos insetos permitem que o ser humano tenha um contato com esses animais nas mais variadas situações, possibilitando experiências particulares que, possivelmente, poderão afetar a percepção do indivíduo em relação a eles (GOODENOUGH, 2003; CAJAIBA; SILVA, 2015). Além disso, essas experiências, aliadas à falta de conhecimento das pessoas em relação aos insetos (COSTA NETO, 2000a; 2000b), podem prejudicar a classificação destes pelos seres humanos, que atribuem a essa classe diferentes grupos taxonômicos, tais como lagartos, serpentes, aves, escorpiões e aranhas (COSTA NETO; MAGALHÃES, 2007), considerando esses animais repugnantes, perigosos e nojentos (SILVA; COSTA-NETO, 2004; ALENCAR et al., 2012).

A percepção, classificação, conhecimento e utilização dos insetos pela população humana são estudados pela Etnoentomologia (POSEY, 1986; COSTA NETO, 2002; 2004). Petiza et al. (2013a, p. 323) definiram Etnoentomologia como "um ramo da etnociência que busca compreender a maneira como diversas culturas percebem, identificam, classificam, nomeiam, utilizam e conhecem o que entendem por ‘inseto' em suas línguas”.

Os estudos etnoentomológicos remontam ao século XIX com a participação de diversos autores no registro das formas de interação entre os seres humanos com os insetos, e como estes são nomeados a partir dos idiomas nativos, pois cada cultura possui diferentes sistemas para realizar a classificação e conceituar os insetos, com base em conhecimentos que foram passados de geração a geração, o que permite a construção de diferentes percepções (POSEY, 1987; CAJAIBA; SILVA, 2017).

Apesar da importância de estudos etnoentomológicos para a compreensão da influência humana sobre os insetos e vice-versa, que permitem a elaboração de novas estratégias de conservação e preservação da biodiversidade e da cultura local (MELO et al., 2015; SANTOS et al., 2015; CARVALHO FILHO, 2017), há poucos trabalhos realizados para o estado do Maranhão (CARVALHO FILHO, 2017). Assim, o presente trabalho tem como objetivo caracterizar as percepções e as atitudes dos moradores do município de Buriticupu sobre os insetos.

\section{Material e Métodos}

A pesquisa foi realizada no município de Buriticupu, localizado na microrregião do Pindaré, no oeste do estado do Maranhão, situada a $430 \mathrm{~km}$ da capital São Luís. O município possui uma população estimada em 71.979 habitantes, segundo dados do Instituto Brasileiro de Geografia e Estatística (IBGE, 2011). O clima predominante é tropical, com temperaturas médias 
anuais variando entre $25^{\circ} \mathrm{C}$ e $27^{\circ} \mathrm{C}$, com dois períodos bem definidos: um chuvoso, de janeiro a junho, e outro seco, correspondente aos meses de julho a dezembro (CORREIA-FILHO, 2011). As principais atividades econômicas são a produção extrativa vegetal, a pecuária e a fruticultura (IBGE, 2011; LIMA et al., 2017).

As coletas foram realizadas por meio de entrevistas semiestruturadas, com base no método de geração de dados (POSEY, 1987), aplicadas a moradores da área urbana do município durante o período de novembro de 2017 a fevereiro de 2018. Foram entrevistadas apenas pessoas com idade igual ou superior a 18 anos. As entrevistas foram gravadas com aparelho MP3, com o consentimento dos informantes, e, posteriormente, foram transcritas com a máxima fidelidade possível e organizadas em um banco de dados padronizado no Microsoft Office Excel $^{\circledR} 2016$ (C) 2016 Microsoft Corporation).

As respostas dos entrevistados foram analisadas segundo o modelo de união das diversas competências individuais (ALVES et al., 2015), em que toda informação pertinente ao assunto pesquisado deve ser considerada. Subsequentemente, as respostas foram analisadas através de estatística descritiva, calculando as frequências relativas das respostas encontradas, sendo os resultados apresentados em porcentagens na forma de tabelas e/ou gráficos (ALVES et al., 2015). Ressalta-se que no que se refere à soma das frequências, em alguns casos, esta ultrapassou o número esperado, pois algumas opiniões dos entrevistados acabaram sendo enquadradas em mais de uma categoria (ALVES et al., 2015). O desenvolvimento do trabalho observou os princípios éticos da pesquisa, em que os entrevistados concordaram em assinar o Termo de Consentimento Livre e Esclarecido (TCLE).

\section{Resultados e Discussão}

Participaram desse estudo 107 pessoas, sendo que 77 eram do sexo feminino (72\%). Do total de entrevistados, $43 \%$ tinham idade entre 18 e 38 anos, $40 \%$ entre 39 e 59 anos e $17 \%$ possuíam idade igual ou superior a 60 anos. A média das idades foi de 42,22 anos (dp $\pm 17,36)$. Quanto à escolaridade dos participantes, 21 pessoas informaram ser analfabetas $(20 \%), 41$ possuíam ensino fundamental incompleto (38\%) e 23 tinham ensino médio completo (21\%).

Com relação ao conhecimento etnobiológico, os entrevistados citaram ao todo 40 tipos de animais; destes, 26 são classificados sistematicamente na classe Insecta (Tabela 1) e 14 são classificados em oito táxons diferentes, entre os quais se destacaram o rato (Muridae), com 36,4\% das citações, seguido por aranha (Araneae) e cobra (Serpentes), com $16,1 \%$ e $15,7 \%$, respectivamente (Tabela 2 ). Os resultados estão de acordo com outras pesquisas etnobiológicas, nas quais é possível observar que as populações classificam, com os insetos, um número considerável de espécies pertencentes a outros táxons (PETIZA et al., 2013a; 2013b). Ulysséa et al. (2010) também registraram resultados semelhantes, em que a aranha, a cobra e o rato tiveram maiores percentuais de citações, sendo classificados como insetos. De acordo com Montenegro et al. (2014), a categoria etnozoológica "inseto" é influenciada pela cultura de cada povo e classificada de acordo com o sentimento interpretado por cada indivíduo, a partir de atitudes mais positivas ou negativas vivenciadas no cotidiano, o que pode explicar a classificação de artrópodes em geral, assim como outros animais, como insetos (BOMFIM et al., 2016). 
TABELA 1: Animais citados pelos moradores de Buriticupu-MA e classificados sistematicamente na classe Insecta.

\begin{tabular}{|c|c|c|c|c|}
\hline Ordem & Nome vulgar & Família/Espécie & $\begin{array}{l}\text { Número de } \\
\text { citações }\end{array}$ & Frequência (\%) \\
\hline \multirow{2}{*}{ Blattodea } & Barata & \multirow{2}{*}{ Blattidae } & 136 & $24,0 \%$ \\
\hline & Cupim & & 6 & $1,1 \%$ \\
\hline \multirow{5}{*}{ Coleoptera } & Besouro/Cascudo & - & 12 & $2,1 \%$ \\
\hline & Rola-bosta & Scarabaeidae & 4 & $0,7 \%$ \\
\hline & Joaninha & Coccinellidae & 4 & $0,7 \%$ \\
\hline & Bicudo & Curculionidae & 1 & $0,2 \%$ \\
\hline & Vagalume & Lampyridae & 1 & $0,2 \%$ \\
\hline \multirow{6}{*}{ Diptera } & Mosquito-da-dengue & Culicidae/Aedes aegypti & 83 & $14,7 \%$ \\
\hline & Mosquito & Simuliidae & 63 & $11,1 \%$ \\
\hline & Muriçoca/Pernilongo & Culicidae & 59 & $10,4 \%$ \\
\hline & Mosca & Muscidae/Musca domestica & 47 & $8,3 \%$ \\
\hline & Mutuca & \multirow{2}{*}{ Tabanidae } & 4 & $0,7 \%$ \\
\hline & Cabo-verde & & 1 & $0,2 \%$ \\
\hline \multirow{4}{*}{ Hemiptera } & Barbeiro & Reduviidae & 13 & $2,3 \%$ \\
\hline & Maria fedorenta & Pentatomidae & 3 & $0,5 \%$ \\
\hline & Percevejo & - & 1 & $0,2 \%$ \\
\hline & Pulgão & Aphididae & 1 & $0,2 \%$ \\
\hline \multirow{3}{*}{ Hymenoptera } & Formiga & Formicidae & 39 & $6,9 \%$ \\
\hline & Abelha & Apoidea & 7 & $1,2 \%$ \\
\hline & Marimbondo & Vespidae & 4 & $0,7 \%$ \\
\hline \multirow{3}{*}{ Lepidoptera } & Lagarta & - & 21 & $3,4 \%$ \\
\hline & Borboleta & - & 8 & $1,4 \%$ \\
\hline & Traça & - & 1 & $0,2 \%$ \\
\hline \multirow{3}{*}{ Orthoptera } & Grilo & Gryllidae & 24 & $4,2 \%$ \\
\hline & Gafanhoto & Acrididae & 12 & $2,1 \%$ \\
\hline & Esperança & Tettigoniidae & 2 & $0,4 \%$ \\
\hline Phthiraptera & Piolho & Pediculidae & 2 & $0,4 \%$ \\
\hline Mantodea & Louva-a-deus & Mantidae & 2 & $0,4 \%$ \\
\hline Siphonaptera & Pulga & - & 5 & $0,9 \%$ \\
\hline
\end{tabular}


TABELA 2: Animais citados pelos moradores de Buriticupu-MA e classificados sistematicamente em outros táxons, diferentes da classe Insecta.

\begin{tabular}{ccccc}
\hline \multirow{2}{*}{ Classe } & Nome vulgar & Ordem/Família & $\begin{array}{c}\text { Número de } \\
\text { citações }\end{array}$ & Frequência (\%) \\
\hline Amphibia & Sapo/Rã & Bufonidae & 9 & $3,7 \%$ \\
\hline \multirow{2}{*}{ Arachnida } & Aranha/Caranguejeira & Araneae & 39 & $16,1 \%$ \\
& Carrapato & Ixodida & 14 & $5,8 \%$ \\
& Escorpião & Scorpiones & 11 & $4,5 \%$ \\
\hline \multirow{2}{*}{ Ave } & Colibri & Trochilidae & 2 & $0,8 \%$ \\
Chilopoda & Corrupião & Icteridae & 1 & $0,4 \%$ \\
\hline Crustacea & Caranguejo & Scolopendromorpha & 17 & $7,0 \%$ \\
\hline Diplopoda & Gangugir/Embuá & Brachyura & 1 & $0,4 \%$ \\
\hline \multirow{2}{*}{ Gastropoda } & Lesma & Julidae & 8 & $3,3 \%$ \\
\hline \multirow{2}{*}{ Mammalia } & Caramujo & Stylommatophora & 2 & $0,8 \%$ \\
& Rato & Pulmonata & 1 & $0,4 \%$ \\
\hline \multirow{2}{*}{ Reptilia } & Morcego & Muridae & 88 & $36,4 \%$ \\
& Lagartixa/Labigó & Chiroptera & 4 & $1,7 \%$ \\
\hline & Lagarto & Serpentes & 38 & $15,7 \%$ \\
\hline
\end{tabular}

Apesar de os informantes citarem animais pertencentes a diversos táxons, a classificação dos insetos segundo a visão acadêmica ainda teve maior frequência de citações em relação aos outros táxons, sendo a barata o inseto mais citado, aparecendo na maioria das respostas $(65 \%)$, seguida por mosquito (31\%), muriçoca/pernilongo (31\%) e mosca (25\%). Modro et al. (2009) também obtiveram, em estudos realizados com docentes e discentes do município de Santa Cruz do Xingu, no Mato Grosso, a maioria dos animais citados pertencentes à Classe Insecta. Resultados semelhantes também apareceram em estudos realizados em uma comunidade rural no semiárido do estado da Paraíba por Montenegro et al. (2014), em que a maioria dos animais citados pelos moradores também eram insetos da classificação lineana.

Outra questão semelhante também foi colocada, porém, esta possuía uma sequência de figuras de 11 animais, em que quatro pertenciam à classe Insecta (barata, grilo, besouros e abelha) e sete eram de diferentes táxons: caracol (molusco), minhoca (anelídeo), caranguejo (crustáceo), lagartixa (réptil), escorpião e aranha (aracnídeos) e lacraia (miriápode). Na questão, pediu-se que os entrevistados observassem as figuras e identificassem aqueles que eram insetos segundo as suas concepções. Dos 107 entrevistados, apenas três $(2,8 \%)$ responderam corretamente, marcando apenas os insetos, enquanto o restante $(97,2 \%)$ marcou animais de outros táxons como sendo pertencentes à classe Insecta (Figura 1). Cajaiba e Silva (2015) em seus estudos constataram que "esse assunto é pouco trabalhado em salas de aulas, e quando trabalhados, são repassados pelos professores de forma superficial". 
FIGURA 1: Percentual dos animais dispostos em figuras marcados como insetos pelos entrevistados.

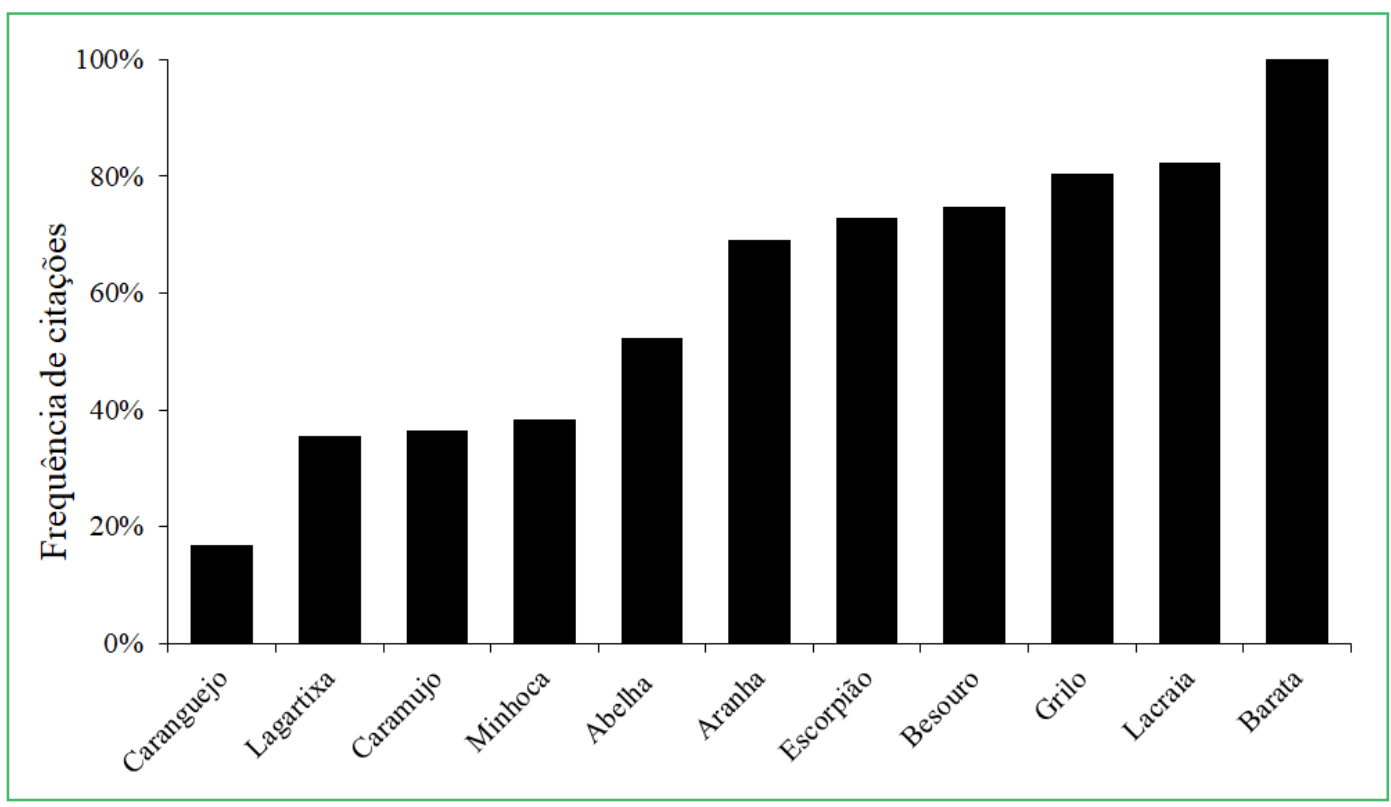

Quando questionados sobre "o que são insetos para você?", a maioria dos entrevistados (68\%) definiu insetos citando exemplos de "animais", mencionando-os como animais pertencentes a outros táxons ou não, como observado em alguns relatos: "É barata, é rato" (E. 1, 48 anos); "É barata, muriçoca, grilo" (E. 2, 38 anos); "Inseto pra mim é aranha, barata, rato" (E. 39, 49 anos). Artrópodes, bem como outros animais, tendem a ser classificados como insetos devido à influência cultural local de cada região estudada, o que interfere na forma de classificação, conceituação e conhecimento sobre a forma de vida dos insetos (PETIZA et al., 2013a; 2013b; MONTENEGRO et al., 2014; BOMFIM et al., 2016).

Alguns entrevistados (7\%) definiram insetos como “coisa nojenta, horrivel". Definições relacionadas à percepção negativa dos insetos também foram encontradas em estudos realizados por Modro et al. (2009) com docentes e discentes do município de Santa Cruz do Xingu, Mato Grosso, em que os discentes definiram os "insetos" como seres pequenos, perigosos ou nojentos. Outras definições como "bicho que transmite doenças" aparecem em 5\% das respostas. Esses tipos de definições afirmam o que foi descrito por Hermogenes et al. (2016) e Bomfim et al. (2016) em seus trabalhos etnoentomológicos, nos quais os entrevistados tendem a construir a etnocategoria "inseto" de acordo com o tipo de experiência negativa vivenciada, ou ainda de acordo com o ambiente inserido e os meios de conhecimentos, ou até mesmo pelas características encontradas em alguns insetos, como a aparência desagradável e a capacidade de transmitir doenças (CAJAIBA; SILVA, 2017). Tal fato pode ser comprovado por meio da questão seguinte, em que os informantes foram perguntados sobre o que pensavam ou sentiam ao ouvir a palavra insetos. Desse modo, palavras como "medo", "pavor" e "nojo" apareceram com maior frequência nas respostas (46,8\%), como demonstram os seguintes relatos: "Coisas que dá nojo" (E. 29, 18 anos); "Inseto é uma coisa nojenta" (E. 21, 30 anos); "É um bicho peçonhento, se não tomar cuidado ele acaba causando irritação" (E. 27, 18 anos, nível de escolaridade: ensino médio completo); "Inseto coisa ruim, o nome fala tudo. Só pra transmitir doenças" (E. 107, 39 anos, nível de escolaridade: ensino médio completo).

Os sentimentos de nojo e medo também foram os mais assinalados pelos moradores da comunidade de Sussuapara em trabalho realizado por Bomfim et al. (2016) e em estudos realizados por Hermogenes et al. (2016) com graduandos da Universidade Federal do Espírito Santo. 
Houve, ainda, os que utilizaram caráter conceitual (2\%) para definir inseto, como: "Uma espécie do reino animal, muito importante na cadeia alimentar e equilíbrio do meio ambiente" (E. 34, 20 anos, nível de escolaridade: ensino médio completo); "Um ser vivo" (E. 55, 24 anos). Apenas 5\% dos entrevistados disseram não saber responder o que é inseto.

As percepções relatadas pelos moradores de Buriticupu foram organizadas em três categorias para facilitar a compreensão de suas opiniões em relação aos seus sentimentos e pensamentos quanto aos animais considerados insetos: sentimentos/pensamentos negativos (77\%); sentimentos/pensamentos neutros (21\%) e sentimentos/pensamentos positivos (2\%). Para os sentimentos/pensamentos neutros, palavras como "nada" e "normal" foram mais comuns para descrever a sensação em relação aos insetos. Já o sentimento/ pensamento positivo pode ser percebido na seguinte frase: "Ele ajuda a combater o mal" (E. 8, 35 anos). Resultados semelhantes podem ser percebidos em estudos realizados por Cajaiba e Silva (2015) e Bomfim et al. (2016). Ao investigar a percepção e o uso dos insetos, pelos moradores da comunidade do Ribeir o da Ilha em Santa Catarina, Ulysséa et al. (2010) também registraram percepções negativas pela maioria dos entrevistados.

Em relação à importância dos insetos para o meio ambiente, $42,1 \%$ dos entrevistados disseram que eles não têm importância nenhuma e 25,2\% expressaram não saber se eles têm ou não alguma importância. Outros $12,1 \%$ informaram que os insetos servem para "combater outros insetos" e 6,5\% afirmaram que estes têm importância para o "equilíbrio do meio ambiente". Esse resultado é semelhante ao encontrado por Bomfim et al. (2016) em uma comunidade rural do cerrado piauiense, onde $40 \%$ dos entrevistados disseram que os insetos não serviam para nada e $4 \%$ informaram que os insetos têm alguma função para o meio ambiente.

Quando questionados sobre "qual o malefício dos insetos para você?", a maioria dos entrevistados $(45,5 \%)$ respondeu que os insetos são transmissores de doenças, e, entre elas, as mais citadas foram: alergia, dengue, coceira, Chikungunya, febre amarela e "lecha".
Animais como ratos, baratas e mosquitos apareceram com maior frequência nas citações dos entrevistados como sendo "insetos" causadores de malefícios, os ratos por transmitirem leptospirose, a barata porque "carrega bactérias e vírus" para "dentro das casas" e os mosquitos por causarem picadas e transmitir doenças como a dengue.

Essa relação entre insetos e transmissão de doenças pode ser percebida na questão seguinte, na qual os entrevistados deveriam responder se conheciam insetos pragas ou transmissores de doenças: $53,7 \%$ dos entrevistados citaram os mosquitos como transmissores de doenças. Desse total, $75,8 \%$ citaram o mosquito da dengue, $10,5 \%$ citaram muriçocas/pernilongos, $8,4 \%$ mencionaram apenas mosquitos (não especificando de qual se tratava), 3,2\% mencionaram o "mosquito da febre amarela" e apenas 2,1\% citaram o "mosquito da malária". O mosquito da dengue (Aedes aegypti) também foi uns dos mais citados em estudos realizados por Carvalho Filho (2017) com moradores do povoado de Bom Sucesso no Maranhão e por Jorge et al. (2014) em um bairro na cidade de Cuiabá, Mato Grosso. Malefícios como "transmissores de doenças" e causadores de reações alérgicas também foram citados em estudos realizados por Hermogenes et al. (2016).

Outros insetos citados como transmissores de doença ou inseto praga foram: barbeiro $(6,8 \%)$, barata $(5,7 \%)$, mosca $(2,8)$ e potó $(2,3 \%)$. A classificação como transmissores de doenças pode estar associada ao fato de a regi o em que se encontra o município ser endêmica da leishmaniose, dengue, Zika e mal ria, uma vez que essas doenças $\mathrm{s}$ o todas transmitidas por insetos. Animais pertencentes a outros táxons também foram incluídos como insetos pragas ou transmissores de doenças, foram eles: rato (13\%), carrapato e lacraia $(1,1 \%)$. Os demais tiveram percentual de $0,6 \%$, como: cobra, aranha, escorpi o e caramujo. O rato também apareceu em estudos realizado por Jorge et al. (2014) como sendo um "inseto" causador de doenças. Essa classificação pode estar relacionada à percepção negativa que as pessoas têm sobre os insetos (MODRO et al., 2009).

Em relação ao conhecimento dos entrevistados sobre como os insetos nascem (origem), $40 \%$ relataram 
não saber, 34\% conseguiram responder corretamente, destes, 26\% responderam que "se originam de ovos" e $8 \%$ informaram que "os insetos vêm de larvas". Foi possível observar respostas com ideias de geração espontânea (16\%), como: "Uns geram de folhas [...]" (E. 7, 59 anos); "Através da água contaminada" (E.10, 41 anos); "Alguns de arroz (gorgulhos)" (E. 76,45 anos). Outros informantes acreditam que os insetos surgem da sujeira ou lixo (6\%). Cajaiba e Silva (2015) encontraram resultados semelhantes ao analisar as percepções dos alunos do ensino fundamental no município de Uruará, Pará, onde a maioria dos discentes não soube responder corretamente sobre a forma de reprodução dos insetos, dizendo que "nascem da goiaba" ou de "animais mortos". Isso pode estar relacionado à falta de conhecimento que ainda prevalece sobre esse assunto e à forma como este é abordado em sala de aula, como descrito por Cajaiba e Silva (2015, p. 124), de forma superficial, o que pode ser relacionado com os $40 \%$ dos entrevistados que disseram não saber como os insetos nascem.

A partir dos resultados obtidos, pode-se afirmar que os moradores do município de Buriticupu categorizam dentro da Classe Insecta outros animais pertencentes a diferentes táxons. O tipo de classificação, conceituação e conhecimento dos entrevistados sobre esses artrópodes estão relacionados com o tipo de sensação ou experiências adquiridas de forma negativa, apesar de alguns ainda reconhecerem os benefícios que estes podem trazer para o ambiente. Isso pode, assim, afetar o comportamento da população a respeito dos insetos, podendo reproduzir medo, nojo ou até mesmo contribuir para a eliminação desses animais. Dessa forma, trabalhos etnoentomológicos tornam-se importantes para compreender a forma como os diferentes povos percebem os insetos. Além disso, podem contribuir para adoção de medidas educativas que venham a possibilitar a sensibilização de diferentes povos sobre a importância dos insetos para o ambiente. Para tanto, é necessária a divulgação desses trabalhos, além do trabalho de assuntos relacionados ao tema no ensino formal e informal de forma a buscar uma mudança na visão negativa relacionada aos insetos.

\section{Referências}

ALENCAR, J. B. R.; SILVA, E. F.; SANTOS, V. M.; SOARES, H. K. L.; LUCENA, R. F. P.; BRITO, C. H. Percepção e uso de "insetos" em duas comunidades rurais no semiárido do estado da Paraíba. Biofar: Revista de Biologia e Farmácia, Campina Grande, v. 9, n. esp., p. 72-91, 2012.

ALVES, M. T. A.; FREIRE, J. E.; BRAGA, P. E. T. O conhecimento local sobre os insetos pelos moradores do município de Groaíras, Ceará. Ensaios e Ciência: Ciências Biológicas, Agrárias e da Saúde, Campo Grande, v. 19, n. 1, p. 7-15, 2015.

BOHAN, D. A.; BOURSAULT, A.; BROOKS, D. R.; PETIT, S. National-scale regulation of the weed seedbank by carabid predators. Journal of Applied Ecology, London, v. 48, n. 4, p. 888-898, 2011.

BOMFIM, B. L. S.; FONSECA FILHO, I. C.; FARIAS, J. C.; FRANÇA, S. M.; BARROS, R. F. M.; SILVA, P. R. R. Etnoentomologia em comunidade rural do cerrado piauiense. Desenvolvimento e Meio Ambiente, Curitiba, v. 39, p. 189-205, 2016.

CAJAIBA, R. L.; PERICO, E.; DALZOCHIO, M. S.; SILVA, W. B.; BASTOS, R.; CABRAL, J. A.; SANTOS, M. G. Does the composition of Scarabaeidae (Coleoptera) communities reflect the extent of land use changes in the Brazilian Amazon?. Ecological Indicators, Amsterdam, v. 74, p. 285-294, $2017 \mathrm{a}$.

CAJAIBA, R. L.; PERICO, E.; SILVA, W. B.; SANTOS, M. Attractiveness of Scarabaeinae (Coleoptera: Scarabaeidae) to different baits in the Brazilian Amazon region. Revista de Biología Tropical, San José, v. 65, n. 3, p. 917-924, 2017 b.

CAJAIBA, R. L.; PERICO, E.; SILVA, W. B.; VIEIRA, T. B.; DALZOCHIO, M. S.; BASTOS, R.; CABRAL, J. A.; SANTOS, $M$. How informative is the response of Ground Beetles (Coleoptera: Carabidae) assemblages to anthropogenic land use changes? Insights for ecological status assessments from a case study in the Neotropics. Science of the Total Environment, Amsterdam, v. 636, p. 1219-1227, 2018.

CAJAIBA, R. L.; SILVA, W. B. Percepção dos alunos do ensino médio sobre os insetos antes e após aulas práticas: um estudo de caso no município de Uruará, Pará. Revista Lugares de Educação, Bananeiras, v. 5, n. 11, p. 118-132, 2015.

CAJAIBA, R. L.; SILVA, W. B. Aulas práticas de Entomologia como mecanismo facilitador no aprendizado de taxonomia para alunos do ensino médio. Scientia Amazonia, Manaus, v. 6, n. 1, p. 107-116, 2017.

CARVALHO FILHO, D. S. Etnoentomologia: a percepção de moradores do povoado de Bom Sucesso, município de Mata Roma, Maranhão, Brasil a respeito dos insetos. 2017. 22 f. Monografia (Graduação em Ciências Biológicas) - Universidade Federal do Maranhão, Chapadinha. 2017.

CORREIA FILHO, F. L.; GOMES, É. R.; NUNES, O. O.; LOPES FILHO, J. B. (Org.). Projeto cadastro de fontes de abastecimento por água subterrânea, estado do Maranhão: relatório diagnóstico do município de Buriticupu. Teresina: CPRM - Serviço Geológico do Brasil, 2011. 31 p.

COSTA NETO, E. M. Conhecimentos e usos tradicionais de recursos faunísticos por uma comunidade Afro-brasileira. 
Resultados preliminares. Interciência, Caracas, v. 25, n. 9, p. 423421. 2000a.

COSTA NETO, E. M. Introdução à etnoentomologia: considerações metodológicas e estudo de casos. Feira de Santana: UEFS, 2000b. 131 p.

COSTA NETO, E. M. Manual de Etnoentomologia. Volume 100. Zaragoza: Editora Zaragoza, Sociedad Entomológica Aragonesa, 2002. 104 p.

COSTA NETO, E. M. Estudos etnoentomológicos no estado da Bahia, Brasil: uma homenagem aos 50 anos do campo de pesquisa. Biotemas, Florianópolis, v. 17, n. 1, p. 117-149, 2004.

COSTA NETO, E. M.; MAGALHÃES, H. F. The ethnocategory "insect" in the conception of the inhabitants of Tapera County, São Gonçalo dos Campos, Bahia, Brazil. Anais da Academia Brasileira de Ciências, Rio de Janeiro, v. 79, n. 2, p. 239-249, 2007.

IBGE. Censo Demográfico 2010, área territorial brasileira. Rio de Janeiro: IBGE, 2011. Disponível em: < https://cidades.ibge.gov. br/brasil/ma/buriticupu>. Acesso em: 9 maio 2018.

GOODENOUGH, W. H. In pursuit of culture. Annual Review of Anthropology, New York, v. 32, n. 1, p. 1-12, 2003.

HERMOGENES, G. C.; LACERDA, F. G.; CARMASSI, G. R.; RODRIGUES, L. N. Percepção entomológica de graduandos da Universidade Federal do Espírito Santo, ES, Brasil. EntomoBrasilis, Vassouras, v. 9, n. 3, p. 180-186, 2016.

HOLANDA-NETO, J. P.; FREITAS, B. M.; BUENO, D. M.; ARAÚJO, Z. B. Low seed/nut productivity in cashew (Anacardium occidentale): effects of self-incompatibility and honey bee (Apis mellifera) behaviour. The Journal of Horticultural Science and Biotechnology, London, v. 77, n. 2, p. 226-231, 2002.

JORGE, V. C.; SÁNCHEZ, D. C. M.; SOUZA, M. D.; PASA, M. C.; REZENDE, E. H. Conhecimentos entomológicos pelos moradores do bairro Verdão em Cuiabá - MT. Enciclopédia Biosfera, Goiânia, v. 10, n. 18, p. 3814-3824, 2014.

LIMA, J. S.; MARTINS, J. S. C.; PEREIRA, K. S.; SOUSA, E. S.; CAJAIBA, R. L. Composição gravimétrica de resíduos sólidos em escolas públicas e privadas no município de Buriticupu, MA. Scientia Amazonia, Manaus, v. 6, n. 3, p. 11-16, 2017.

MELO, A. C. B.; ARAÚJO, F. L.; FREIRE, J. E.; BRAGA, P. E. T. O conhecimento popular acerca dos insetos no município de Cariré, Ceará, Brasil. Ciência e Natura, Santa Maria, v. 37, n. 2, p. 253260, 2015.

MODRO, A. F. H.; COSTA, M. S.; MAIA, E.; ABURAYA, F. H. Percepção entomológica por docentes e discentes do município de Santa Cruz do Xingu, Mato Grosso, Brasil. Biotemas, Florianópolis, v. 22, n. 2, p. 153-159, 2009.
MONTENEGRO, I. F.; ALENCAR, J. B. R.; SILVA, E.F.; LUCENA, R. F. P. L.; BRITO, C. H. Conhecimento, percepção e uso de animais categorizados como "insetos" em uma comunidade rural no semiárido do estado da Paraíba, Nordeste do Brasil. Gaia Scientia, João Pessoa, v. 8, n. 2, p. 250-270, 2014.

NITZU, E.; NAE, A.; POPA, I. The fauna of soil beetles (Edaphic Coleoptera) as a sensitive indicator of evolution and conservation of ecosystems. A study on the altitudinal gradient in the Rodnei Mountains Biosphere Reserve (the Carpathians). Monographs, Viena, v. 12, p. 405-416, 2008.

PETIZA, S.; HAMADA, N.; BRUNO, A. C.; COSTA NETO, E. M. Etnoentomología Baniwa. Boletín de la Sociedad Entomológica Aragonesa, Zaragoza, n. 52, p. 323-343, 2013 a.

PETIZA, S.; HAMADA, N.; BRUNO, A. C.; COSTA NETO, E. M. Etnotaxonomia entomológica baniwa na cidade de São Gabriel da Cachoeira, estado do Amazonas, Brasil. Revista de Antropologia, Belém, v. 5, n. 3, p. 708-732, 2013 b.

POSEY, D. A. Entomologia de tribos indígenas da Amazônia. In: RIBEIRO, D. (Ed.). Suma etnológica brasileira. Vol. 1. Rio de Janeiro: Petrópolis Vozes/Finep, 1986. p. 251-272.

POSEY D. A. Temas e inquirições em etnoentomologia: algumas sugestões quanto à geração de hipóteses. Boletim Museu Paraense Emílio Goeldi, Belém, v. 3, n. 2, p. 99-134, 1987.

QODRI, A.; RAFIUDIN, R.; MOEDJITO, W. A. Diversity and abundance of Carabidae and Staphylinidae (Insecta: Coleoptera) in four montane habitat types on Mt. Bawakaraeng, South Sulawesi. Hayati Journal of Biosciences, Amsterdam, v. 23, n. 1, p. 22-28, 2016.

SANTOS, J. R. L.; THOMAS, S. E. O.; DORVAL, A.; PASA, M. C. A etnoentomologia na comunidade Mata Cavalo de Baixo em Nossa Senhora do Livramento, MT, Brasil. Biodiversidade, Rondonópolis, v. 14, n. 2, p. 84-92, 2015.

SILVA, T. F. P.; COSTA NETO; E. M. Percepção de insetos por moradores da comunidade Olhos D’Água, município de Cabaceiras do Paraguaçu, Bahia, Brasil. Boletín de La Sociedad Entomológica Aragonesa, Zaragoza, v. 35, n. 1, p. 261-268, 2004.

ULYSSÉA, M. A.; HANAZAKI, N.; LOPES, B. C. Percepção e uso dos insetos pelos moradores da comunidade de Ribeirão da Ilha, Santa Catarina, Brasil. Biotemas, Florianópolis, v. 23, n. 3, p. 1191-202, 2010. 